\title{
Interplay of Rearrangements, Strain, and Local Structure during Avalanche Propagation
}

\author{
Ge Zhang $\odot$, Sean A. Ridout $\odot$, and Andrea J. Liu $\odot^{*}$ \\ Department of Physics and Astronomy, University of Pennsylvania, \\ Philadelphia, Pennsylvania 19104, USA
}

\begin{abstract}
(Received 10 August 2020; revised 29 July 2021; accepted 10 August 2021; published 27 October 2021)
Jammed soft disks exhibit avalanches of particle rearrangements under quasistatic shear. We introduce a framework for understanding the statistics of the progression of avalanches. We follow the avalanches (simulated using steepest descent energy minimization) to decompose them into individual localized rearrangements. We characterize the local structural environment of each particle by a machine-learned quantity, softness, designed to be highly correlated with rearrangements, and analyze the interplay between softness, rearrangements, and strain. Local yield strain has long been incorporated into elastoplastic models; here we show that softness provides a useful proxy for local yield strain. Our findings demonstrate that elastoplastic models must take into account the fully tensorial strain field in order to include the effects of changes in local yield strain due to rearrangements and introduce the equations underpinning a structuroelastoplastic model that includes local softness.
\end{abstract}

DOI: 10.1103/PhysRevX.11.041019

Subject Areas: Materials Science, Soft Matter, Statistical Physics

\section{INTRODUCTION}

All disordered solids respond elastically at low strain but flow plastically at sufficiently high strain. As strain increases beyond the elastic regime, disordered solids partially relax via intermittent localized rearrangements until they reach the yield strain, where they begin to flow. Up to the yield strain, disordered solids display surprisingly universal behavior with yield strains quite tightly distributed around 3\% for systems ranging from metallic and molecular glasses to nanoparticle, colloidal and granular packings, and with rearrangements localized on the scale of the constituent particle size [1]. Beyond the yield strain, however, disordered solids exhibit several different classes of plastic behavior. Foams can flow indefinitely via localized rearrangements without ever fracturing [2] (ductile behavior). Many other ductile systems exhibit crackling noise or avalanche behavior [3-6], while brittle systems typically exhibit shear banding and brittle fracture [7]. Here we focus on avalanche behavior.

An avalanche consists of a series of localized rearrangements. Avalanches in driven disordered solids have been studied in numerous experiments and simulations, including Refs. [8-11]. A class of models known as elastoplastic

\footnotetext{
*ajliu@physics.upenn.edu
}

Published by the American Physical Society under the terms of the Creative Commons Attribution 4.0 International license. Further distribution of this work must maintain attribution to the author(s) and the published article's title, journal citation, and DOI. models describes such avalanches in terms of the interplay of rearrangements and elastic stress [12]. In such models, a local yield strain or stress is assigned to each site, an increase of elastic stress can cause a local region to yield and rearrange, while conversely, a local rearrangement can increase stress elsewhere. A typical elastoplastic model subjected to $x y$-shear strain is summarized as a flow chart in Fig. 1(a). It has become increasingly clear, however, that rearrangements and elasticity do not tell the whole story. Systems with identical microscopic interactions can show ductile or brittle behavior depending on preparation history $[13,14]$. This has been taken into account in elastoplastic models by varying the local yield strain distribution by hand [15], but a more fundamental approach would take local structure into account. This is done by phenomenological theories that postulate structural defects prone to rearrange [16,17], but an alternate approach, which we adopt here, is to generalize elastoplastic models to take local structure into account. The first step in this approach is to elucidate the connection between local structure and the physics included in elastoplasticity models. While it has been shown that certain local structural environments are much more likely to rearrange than others [18-21], effects of rearrangements on local structure have not been established, even though it is clear that they must exist. It is also clear that elastic stresses can distort the structural environment surrounding a particle [1]. These considerations point to the need for detailed understanding of the interplay of local structure, rearrangements, and elasticity.

In this paper, we go back to basics to untangle the interplay of local structure, rearrangements, and strain in 


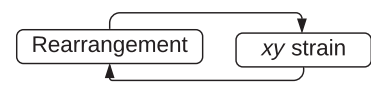

(a)

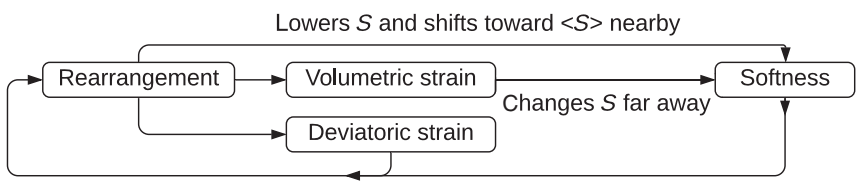

(b)

FIG. 1. (a) In a typical elastoplastic model under an $x y$-shear strain, rearrangements give rise to $x y$ strain and that strain can trigger regions of low local yield strain to rearrange. This interplay gives rise to rearrangement avalanches. (b) Summary of the interplay between rearrangements, strain, and softness (local structure) in our augmented structuro-elastoplasticity (STEP) model. A rearrangement decreases the softness of nearby particles, alters the softness of far-away particles through volumetric strain, and exerts a deviatoric shear strain on all particles. Softness determines the local yield strain, and the local deviatoric strain can trigger regions of high softness (low local yield strain) to rearrange, giving rise to rearrangement avalanches.

athermal, quasistatically sheared jammed packings of soft disks. While some aspects of this interplay have been understood for a long time, such as quadrupolar strain fields arising from rearrangements, a full analysis that includes local structure has not been carried out before. Our analysis leads to a "structuro-elastoplasticity" (STEP) framework for avalanches in disordered solids. In brief, as we demonstrate in this paper, the steps that allow construction of a structuro-elastoplasticity model for a given system are as follow. (1) We perform particle-based simulations detailed in Sec. II A, identifying rearrangements by calculating nonaffine deformation around each particle (Sec. II B). (2) We then describe local structure with a machinelearned quantity, softness $[1,20,22,23]$, in Sec. II C. Softness has been shown to provide useful insight into the dynamics of supercooled liquids and glasses [20,22,24] and has been demonstrated to be predictive of rearrangements in athermal, quasistatically sheared amorphous solids [21]. Following this approach [20], we describe softness as the weighted sum of a set of structural quantities based on the local pair correlation function, where the weights are chosen to maximize the correlation with rearrangements that occur during avalanches. (3) We then study the strain field caused by rearrangements in Sec. III A. This strain field can be decomposed into deviatoric and volumetric parts, which have distinct roles in the avalanche process. We demonstrate that the deviatoric part triggers new rearrangements (Sec. III B), while the volumetric part affects the softness field (Sec. III C). (4) Lastly, we study how softness and deviatoric strain work together to create more rearrangements in Sec. III D.

The resulting STEP model for a jammed system of Hertzian disks under athermal quasistatic shear is shown in
Fig. 1(b). This model is richer than a standard elastoplastic model shown in Fig. 1(a). For this system, we find that rearrangements give rise to volumetric strain that increases softness far from the rearrangement. The effects of volumetric strain are not typically included in elastoplastic models, but here we find that it plays an important role. At the same time, we find that rearrangements scramble the structure nearby in a way that lowers softness nearby and shifts it toward the mean softness. Finally, rearrangements give rise to a deviatoric strain, which pushes particles of high softness, which have lower yield strains, beyond their yield strains so that they rearrange. Elastoplastic models generally assume that only the $x y$ strain pushes particles beyond their yield strains for systems subjected to $x y$ shear; our results show that all parts of the full tensorial strain may play different roles in the avalanche process.

\section{NUMERICAL DETAILS}

\section{A. Simulations}

We generate two-dimensional packings of $N$ soft disks in a simulation box with periodic boundary conditions. The disks interact with each other through the pairwise additive Hertzian potential:

$$
u_{2}(r)= \begin{cases}\left(1-\frac{r}{\sigma_{i}+\sigma_{j}}\right)^{2.5} & \text { if } r<\sigma_{i}+\sigma_{j} \\ 0 & \text { otherwise, }\end{cases}
$$

where $\sigma_{i}$ is the radius of the $i$ th disk. To avoid crystallization, we use a $1: 1$ mixture of particles with $\sigma=0.5$ and $\sigma=0.7$. We adjust the system size $V$ so that the packing fraction $\phi=\sum_{i} \pi \sigma_{i}^{2} / V$ is 0.9 .

Starting from random initial conditions, we minimize the potential energy to find the initial zero-temperature jammed state. We then repeatedly apply a small shear-strain step of $\delta \epsilon$, minimizing the energy after each step, until the total strain reaches $\epsilon_{\text {end }}$. The stress-strain relation for a single configuration, shown in Fig. 2, confirms the existence of avalanches. We generated 5 trajectories with $N=10^{5}$, $\delta \epsilon=10^{-5}$, and $\epsilon_{\text {end }}=0.1$; and 20 trajectories with $N=4000, \delta \epsilon=10^{-4}$, and $\epsilon_{\text {end }}=2$. This smaller system with $N=4000$ is shown in Fig. 2 for visual clarity. It is also used to train the machine-learning algorithm because we need to access larger shear strains, as detailed in the Supplemental Material [25]. All of the remaining analysis was carried out on the larger system.

It is well known [26] that during athermal quasistatic shear, energy drops mark rearrangements that can be either localized or extended due to avalanches. In each step of strain followed by energy minimization, we calculate the final energy to monitor for energy drops. As detailed in the Supplemental Material [25], we use steepest descent to accurately simulate the overdamped relaxation process from the beginning of the energy drop to the end. 

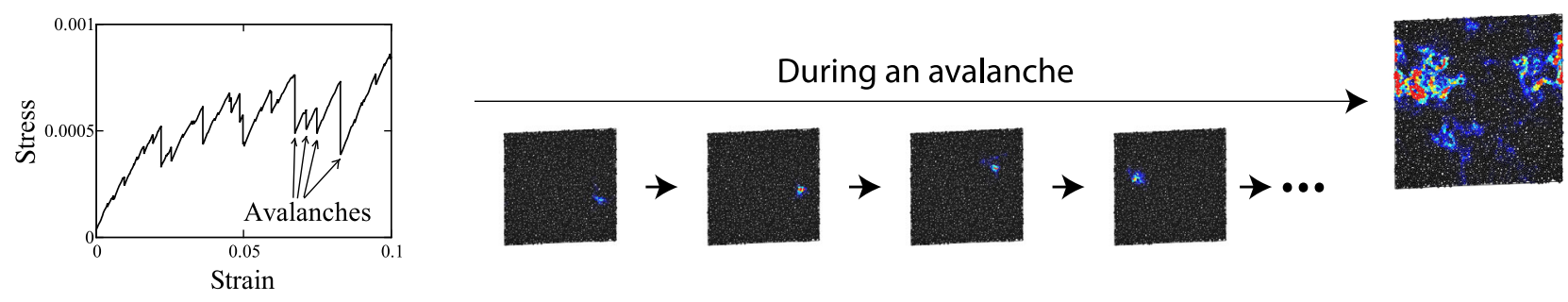

FIG. 2. As strain increases, avalanches occur during stress drops. During an avalanche, some constituent particles rearrange, triggering other localized rearrangements far away in the depicted system of $N=4000$ particles. Here, the nonaffine displacement $D_{\text {min }}^{2}$ of particles is represented on a black-to-blue-to-red scale with red corresponding to high values of $D_{\min }^{2}$. The rightmost plot depicts the cumulative $D_{\min }^{2}$ measured over the entire stress drop.

The step size is adjusted on the fly to balance accuracy and computational cost. During the energy minimization, we save intermediate configurations that are equidistant in configuration space; more specifically, the sum over particles of particle displacement squared, $\sqrt{\sum_{i}^{N} \delta \mathbf{r}_{i}^{2}}$, is chosen to be 0.15 between successive frames. This choice is made so that movies generated from such frames are smooth (see the movie in Supplemental Material). Since we use overdamped dynamics, we can define "time" as the step size divided by the gradient of the potential energy. With this definition, the distribution of time intervals between frames is shown in Fig. S1 [25].

For comparison, we also saved intermediate configurations spaced according to a fixed decrease of energy or fixed time elapsed. However, these schemes resulted in an uneven distribution of $D_{\min }^{2}$ along the trajectory. More specifically, we find that the distributions of $D_{\min }^{2}$ for the first and second halves of avalanches are the same for the first sampling scheme but not for the latter two. Since an even distribution of $D_{\min }^{2}$ is important for training the machine-learning algorithm, we chose the first sampling scheme.

\section{B. Identifying rearrangers}

To identify rearranging particles, or "rearrangers," we calculate $D_{\min }^{2}[27]$ :

$$
D_{\min }^{2}(k)=\frac{1}{M_{k}} \sum_{i}^{M_{k}}\left[\mathbf{r}_{i k}^{\prime}-\mathbf{J}_{k} \mathbf{r}_{i k}\right]^{2},
$$

where the sum is over all neighbors of particle $k$ within a distance of $R_{D}=2$. Here $M_{k}$ is the number of such neighbors, $\mathbf{r}_{i k}$ and $\mathbf{r}_{i k}^{\prime}$ are the vector separations between particles $i$ and $k$ at two consecutive frames, respectively, and $\mathbf{J}_{k}$ is the "best-fit" local deformation gradient tensor about particle $k$ that minimizes $D_{\min }^{2}$. We will later extract three different strain components near each particle $k$ from $\mathbf{J}_{k}$, including the volumetric (isotropic) strain $k=[\operatorname{Tr}(\mathbf{J})-2] / 2$, total deviatoric strain $\tilde{\epsilon}=\left|\lambda_{1}-\lambda_{2}\right|$, and shear strain in the $x y$ direction (the direction of the global shear), $\epsilon_{x y}=\left[\mathbf{J}_{12}+\mathbf{J}_{21}\right] / 2$, where $\lambda_{1}$ and $\lambda_{2}$ are eigenvalues of $\left(\mathbf{J}+\mathbf{J}^{T}\right) / 2$, where $\mathbf{J}^{T}$ is the transpose of $\mathbf{J}$. The strain field far away from a rearranger is qualitatively insensitive to the choice of the cutoff distance $R_{D}$. We chose $R_{D}=2$ because for smaller $R_{D}$ the fitting to a local affine-deformation tensor $\mathbf{J}_{k}$ occasionally fails, while for larger $R_{D}$ the near-field strain field is smeared. A particle with $D_{\min }^{2}$ above a certain threshold, $d_{\text {soft,small }}=0.0025$ for small particles and $d_{\text {soft,large }}=0.0015$ for large particles, is a rearranger. The rest of the paper presents results for rearrangers that are small particles in our binary mixture, but we have verified that results for large-particle rearrangers are qualitatively the same. When studying the strain and softness change caused by a rearrangement at a large distance [Figs. 2, 5, and 6(b), but not Fig. 6(a) because it is not necessary], we focus on frames that (1) contain only one rearranger and (2) contain no particle that is not close to the rearranger (distance greater than 5) that has $D_{\min }^{2}>0.01 d_{\text {soft }}$. The latter criterion is introduced to exclude frames with multiple rearrangements.

\section{Calculating softness}

Following previous work [20], we calculate softness using the support-vector machine algorithm with a linear kernel. Briefly, we characterize the local structural environment by a set of scalar variables, where each variable corresponds to a function that depends on the structure of a particle's neighborhood. We construct a high-dimensional space in which each orthogonal axis corresponds to a different structure function so that the structure of the neighborhood of a particle is described by a point in this space. We then select a training set consisting of two subsets-particles that are rearranging and particles that are not rearranging - and find the points in the high-dimensional space for each of these particles. We use the supportvector machine algorithm to construct the coefficients of the linear combination of structure functions that is normal to the hyperplane that best separates the two training sets. This linear combination is what we call the "softness"; the linear combination can be used to calculate the softness of each particle as a function of time during the relaxation process following an avalanche. 
To select the training set, we identify 7500 rearranging particles with $D_{\min }^{2}>d_{\text {soft }}$ between two adjacent frames during energy minimization and 7500 nonrearranging particles with $D_{\min }^{2}<d_{\text {hard }}$ between two energy-minimized frames separated by a shearing strain of $\delta \epsilon$. We use two sets of parameters listed in Table I of the Supplemental Material [25].

For a good training set we need nonrearranging particles that do not rearrange over a long period of time prior. To obtain such particles, we simulated smaller systems over a longer shear strain window. Specifically, we generated 20 trajectories with $N=4000, \delta \epsilon=10^{-4}$, and $\epsilon_{\text {end }}=2$. After training, we verified that the softness distribution $P(S)$ and the softness distribution for rearrangers $P(S \mid R)$ are nearly the same, and that the probability that a particle with a given softness is rearranging, $P_{R}(S)$, is very similar for the two system sizes (see Fig. S2 of the Supplemental Material [25]).

We must also choose structure functions to characterize softness. Although previous work employed two-body as well as three-body structure functions, we found that the three-body ones are computationally expensive and contribute less than $1 \%$ increase in the accuracy, so we neglected them [20]. To further improve computational efficiency, we use linear two-body structure functions:

$$
G_{m}(i)=\sum_{j} g_{m, i j}
$$

where

$g_{m, i j}=\left\{\begin{array}{cc}1-\left(r_{i j}-r_{m}\right) /\left(r_{m-1}-r_{m}\right) & \text { if } r_{m-1}<r<r_{m} \\ 1-\left(r_{i j}-r_{m}\right) /\left(r_{m+1}-r_{m}\right) & \text { if } r_{m}<r<r_{m+1} \\ 0 & \text { otherwise, }\end{array}\right.$

and $r_{m}$ is the location of the $m$ th radial function. The training and testing accuracy is the same for these structure functions as for the standard Behler-Parrinello structure functions with Gaussian smoothing [28]. We use multiple sets of $r_{m}$ listed in Table II of the Supplemental Material [25].

Finally, we adopt the ensemble method to calculate softness: we train multiple hyperplanes and average their predictions. For each combination of training set (Table 1 of the Supplemental Material [25]) and structure function placement (Table 2 of the Supplemental Material), we train 5 hyperplanes. This yields 60 hyperplanes (per species) in total. The validation accuracy for individual hyperplanes varies in a small range of 0.878-0.926. The final softness of a particle is the average of the signed distance to all hyperplanes.

In summary, the softness of particle $i$ is essentially a weighted integral over the local pair correlation function $g_{i}(r)$. The weight function is inferred by the linear support vector machine to maximize the accuracy of predicting rearrangers. As in Ref. [20], the weighting is highly negative at the first peak of $g(r)$, implying that particles with fewer neighbors have higher softness, consistent with intuition based on the cage picture. Softness and rearrangements are strongly correlated, as we show in Fig. 5(b), but are distinct concepts. Softness is a structural quantity while rearrangements are dynamical objects.

\section{DECONSTRUCTING THE AVALANCHE PROCESS}

In Fig. 2 and the video in Supplemental Material [25], we confirm that during avalanches, rearrangements are indeed localized and sequential, as assumed in elastoplastic models [12]. Moreover, consecutive rearrangements can be very far apart. In this section, we study the interplay of rearrangements, softness, and elasticity piece by piece, first examining the effects of rearrangements on strain in Sec. III A, then the effects of strain on rearrangements in Sec. III B, and the effects of rearrangements and their resulting strain fields on softness in Sec. III C, and the effects of strain and softness on rearrangements in Sec. III D. Our results in this section are summarized in Fig. 1(b).

\section{A. Strain field due to rearrangement}

We begin by examining the effect of a rearrangement at the origin on the strain at $\mathbf{r}$, averaged over many rearrangements.

The near-field behaviors of the local strains depend on microscopic details of how rearrangements locally deform their surroundings, but in the far field we expect the local strains to be well described by elasticity theory. In the far field, one typically approximates the rearrangement as a point plastic shear strain, equivalent to a pair of point force dipoles. The dipole can have any orientation in a disordered system, but is not isotropically distributed due to the global shear breaking rotational symmetry. The responses to this source at position $\mathbf{r}$ and time $t$ following a rearrangement at the origin at $t=0$ are given in Eqs. (A5)-(A7). As we show in Appendix B, the shear strain source due to the rearrangement is very long-lived, so the response to the point plastic shear strain is well approximated by the infinite-time limit, shown in Eq. (A8). Specifically, $\epsilon_{x y}$ has an $r^{-2}$ radial dependence and a quadrupolar angular dependence (bottom row in Fig. 3). This is consistent with previous analytical derivations [29], numerical measurements [9,26,30], and experiments [9,31]. The deviatoric strain $\tilde{\epsilon}$ (middle row in Fig. 3) likewise decays as $r^{-2}$ (red solid line in left-hand plot) but with an isotropic angular dependence (right-hand plot), as expected from continuum elasticity (see the Appendix A). The existence of this strain field arising from the rearrangement is represented by the arrow connecting "Rearrangements" to "Deviatoric strain" in Fig. 1(b).

The volumetric strain $k(r)$ is typically neglected in systems of fixed total volume, but as we will show, it 

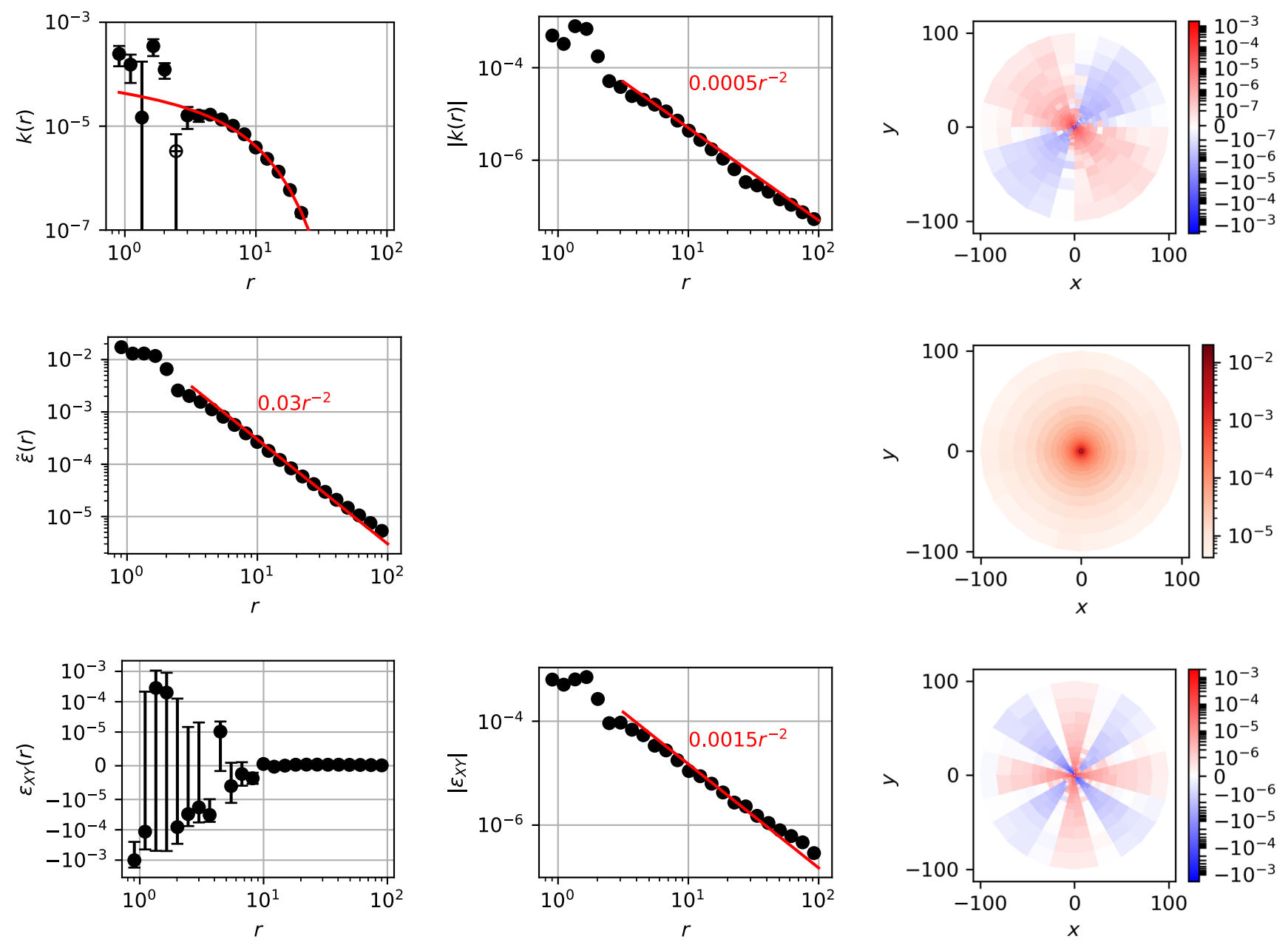

FIG. 3. Mean volumetric strain $k$ (top row), mean deviatoric strain $\tilde{\epsilon}$ (middle), and mean shear strain in the $x y$ direction (the direction of the global shear) $\epsilon_{x y}$ (bottom) per frame caused by a rearranging particle at the origin. Angular-averaged (left-hand column), angularaveraged absolute value (middle), and angular (right) versions are shown. Note that the middle row and column plot is not shown because $\tilde{\epsilon}$ is always non-negative. In the top left-hand plot, solid circles represent positive values of $k(r)$, while open circles represent negative values. Red lines are fits to continuum-elasticity predictions detailed in the text and Appendixes [Appendix A for $|k(r)|, \tilde{\varepsilon}(r)$, and $\left|\varepsilon_{x y}(r)\right|$; and Appendix B for $\left.k(r)\right]$.

plays an important role because softness is strongly dependent on local density. It is the sum of two terms. The first term is the volumetric strain in response to a shear strain source, given in Eq. (A5). This is a $\sin (2 \theta) r^{-2}$ term that dominates in the top middle and top right-hand plots of Fig. 3. The second term is the effect of a point-compression source since the rearrangement can also give rise to local plastic compression. This point compression causes the surroundings to dilate $(k>0)$. This has a transient effect since the total volume of the system is conserved, but is significant because it gives rise to a contribution to $k(r)$ [Eq. (A9)] that does not angle average to zero. The top lefthand plot of Fig. 3 shows that the angular-averaged volumetric strain $k(r)$ is positive at most $r$ and does not exhibit a power-law decay. As we detail in Appendix B, the shape of this curve can be explained by the convolution of a finite-time elastic kernel and a point-compression source with Gaussian time dependence (red solid curve). Reference [32] also appears to provide evidence of local dilation in the strain field due to a rearranger. These results are represented by the arrow connecting "Rearrangement" to "Volumetric strain" in Fig. 1(b).

Although the results shown here are for two-dimensional systems, we have confirmed that the expected scalings for volumetric and deviatoric strain are observed in three dimensions [25], providing strong evidence in favor of our interpretation of the roles of volumetric, deviatoric, and $x y$ strain.

We next show that deviatoric and volumetric parts have distinct roles in the avalanche process. The deviatoric strain triggers new rearrangements (Sec. III B), while the volumetric strain affects the softness field (Sec. III C). 


\section{B. Strain field triggering rearrangements}

We now turn to the effect of the induced strain on the next rearrangement. In elastoplastic models, it is typically assumed that it is the $x y$ component of strain due to a rearrangement triggers other rearrangements in a system subjected to an externally applied $x y$ strain [12]. To test this, we first compute the frame-dependent pair correlation function of rearrangers $g_{2}(\mathbf{r}, \delta f)$, namely the probability of finding a rearrangement at $\mathbf{r}$ after $\delta f$ frames, given a rearrangement at the origin at frame $\delta f=0$. Results for several values of $\delta f$ are presented in Fig. 4.

We first focus on the temporal dependence. As $\delta f$ increases, the rearranger pair correlation function $g_{2}(r, \delta f)$ for $r \lesssim 5$ decreases while that for $r \gtrsim 5$ increases. This occurs because the probability that a rearrangement will jump to a distant location increases with time (as measured in frames). The evolution with the number of frames reflects the course of the avalanche due to propagation of the strain induced by a rearrangement, which alters softness and can trigger further rearrangements.

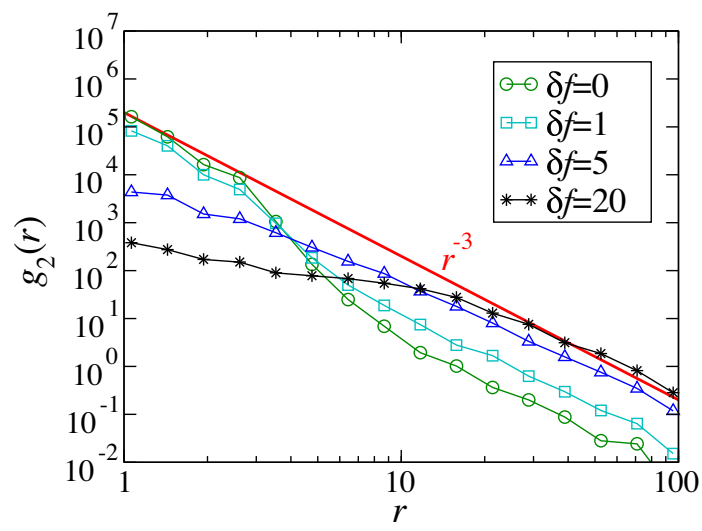

(a)

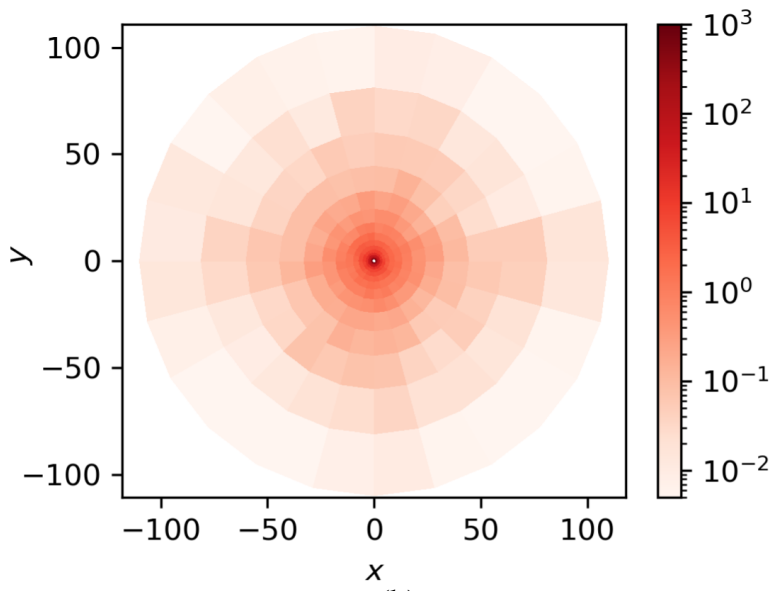

(b)

FIG. 4. (a) The time-dependent pair correlation function of rearrangers $g_{2}(r, \delta f)$ for different numbers of frames $\delta f$ following the rearrangement at the origin at frame $f=0$. (b) The timeaveraged directional plot $g_{2}(\mathbf{r})=(1 / F) \sum_{\delta f=0}^{F} g_{2}(\mathbf{r}, \delta f)$, where $F=20$.
Radially, $g_{2}$ decays approximately as $r^{-3}$ for sufficiently large $r$, independent of $\delta f$. This is consistent with either $\tilde{\epsilon}$ or $\epsilon_{x y}$, which both decay as $r^{-2}$, due to the following argument. Two earlier studies of systems with spherically symmetric potentials found that the cumulative distribution of the local yield strain has a low-yield-strain tail described by a power law with exponent $1.6[33,34]$. On general grounds this scaling should also apply to our system [33], so the probability that a rearrangement is triggered by $\tilde{\epsilon}$ or $\epsilon_{x y} \sim r^{-2}$ should scale as $\left(r^{-2}\right)^{1.6}=r^{-3.2}$, roughly consistent with the scaling we observe in $g_{2}$.

The angular dependence of $g_{2}(\mathbf{r})$ is nearly isotropic and clearly does not show a quadrupolar dependence. This is

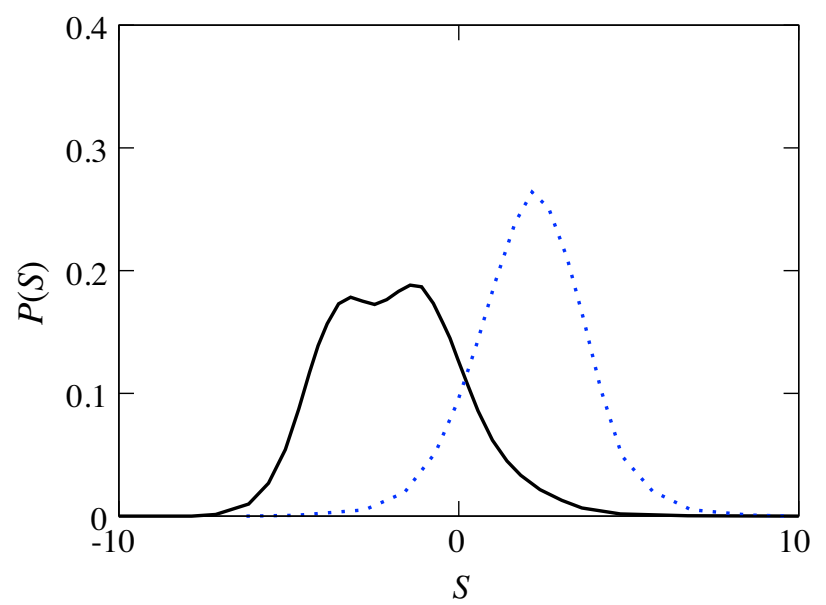

(a)

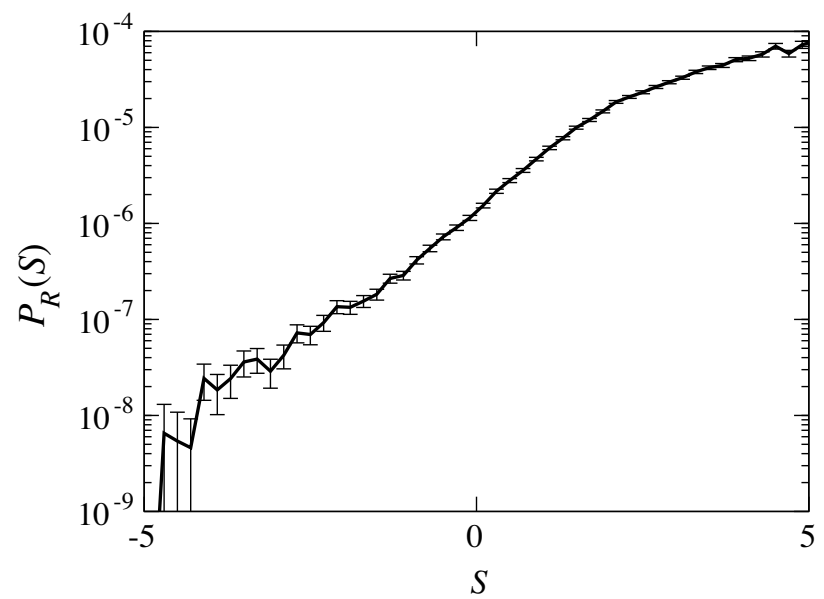

(b)

FIG. 5. Performance of machine-learned softness. (a) The distribution of softness for all particles (black solid curve) and for rearrangers only (blue dotted curve). There is a pronounced difference between the two distributions. The two peaks in the black solid curve come from particles with four and five neighbors, respectively, as we demonstrate in the Supplemental Material [25]. (b) The probability that a particle is rearranging $P_{R}$ as a function of its softness. As the softness increases, $P_{R}$ increases by 4 orders of magnitude, verifying the high correlation between softness and rearrangements. 
consistent with the angular dependence of $\tilde{\epsilon}$, not $\epsilon_{x y}$ (see Fig. 3). We therefore conclude that rearrangement-induced shear strain in any direction can trigger rearrangements equally well. This result contradicts the assumption of many elastoplastic models that $\epsilon_{x y}$ is solely responsible for triggering rearrangements. Such an assumption might be a good approximation in shear-banding systems, where anisotropic (quadrupolar) rearranger pair correlation functions have been observed [9,32,35], but is not valid for the ductile system studied here. A recent elastoplastic model takes into account the entire strain tensor [10]. Our result justifies such an approach.

In short, the results of this section show that it is deviatoric strain $\tilde{\epsilon}$ that is responsible for triggering rearrangements, justifying the arrow connecting "Deviatoric strain" to "Rearrangements" in Fig. 1(b).

\section{Effects of rearrangements and strain on softness}

In training the machine-learning algorithm to obtain softness, we find that $90 \%$ of rearrangers have $S>0$, while $84 \%$ of nonrearrangers have $S<0$. Moreover, Fig. 5 shows that the softness distribution for rearrangers is very different from that of the whole population, and that the probability that a particle rearranges increases by 4 orders of magnitude as softness increases. These results verify that softness is strongly correlated with the propensity to rearrange. These results establish the arrow connecting "Softness" to "Rearrangements" in Fig. 1(b).

In turn, rearrangements can affect softness. We find that the average difference in softness of a rearranger immediately before and after the rearrangement is $\langle\Delta S\rangle_{R}=-0.75$; the softness of a rearranger drops significantly when it rearranges. Rearrangements can also affect the softness of particles elsewhere; we plot the mean softness change $\Delta S(\mathbf{r})$ of a particle at $\mathbf{r}$ due to a rearrangement at the origin in Fig. 6. Rearrangements make overlapping neighbors $(r<1)$ softer and noncontacting nearby particles $(1<r<5)$ less soft. Rearrangements also make distant particles $(r>5)$ softer or harder depending on the orientation. The distance and angular dependences of the far-field $\Delta S$ are consistent with the volumetric strain $k$ (see Fig. 3), suggesting that it is caused by $k$. This is not surprising since softness is highly sensitive to density. This result establishes the arrow connecting "Volumetric strain" to "Softness" in Fig. 1(b).

To understand the near-field effect of rearrangements on softness, we first note that in a thermal Lennard-Jones system, the mean softness of nonrearranging particles with a given initial softness $S_{0}$ evolves toward its mean value for any $S_{0}$ [20] due to rearrangements of other particles. Here we ask if the same effect exists in our quasistatically sheared system. For particles within a short distance $r \leq 1.6$ to a rearranger, we plot the softness change versus the original softness and perform a linear fit, presented in Fig. 7(a). We plot the slopes $c_{1}(r)$ of such fits at several different $r$ in Fig. 7(b). For $r<10$ and $r>30, c_{1}$ is negative, indicating that softness in our system also has the tendency to approach its mean at these distances. However, $c_{1}$ is positive for $10<r<30$, suggesting the opposite effect. The effect is small and negligible, and is probably because softness tends to increase in this range of $r$ [see Fig. 6(a)], and the softer a particle is, the floppier its local environment is, and the more tendency it has to deform, even if such deformation generally raises $S$. More important is the magnitude of $c_{1}(r)$ : we see that the magnitude of $c_{1}(r)$ decays rapidly with $r$ and is well described as a power law: $\left|c_{1}(r)\right|=0.06 r^{-3.2}$. Finally, $c_{1}(r)$ appears to be independent of the angle $\theta$.

Overall, our results suggest that the mean softness change of a particle with softness $S$ at $\mathbf{r}$ when a particle at the origin rearranges is

$$
\Delta S(\mathbf{r}, S)=c_{0}(r)+c_{1}(r)(S-\langle S\rangle)+b k(\mathbf{r}),
$$

where $c_{1}(r)$ is given in Fig. 7(b), and $b \approx 207$. To find $c_{0}$, we subtract $b k(\mathbf{r})$ from $\Delta S(\mathbf{r})$. Similar to $c_{1}$, we do not find any angular dependence in $c_{0}$. We plot its $r$ dependence in Fig. 7(b). Clearly, $c_{0}$ and $c_{1}$ exhibit similar power-law decays; we find $\left|c_{0}(r)\right|=0.3 r^{-3.1}$. With the fit, Eq. (5)

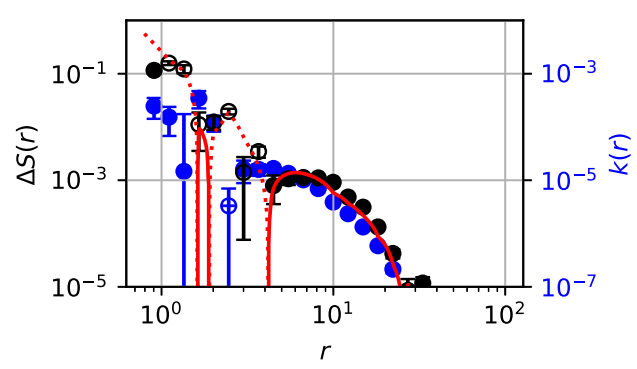

(a)

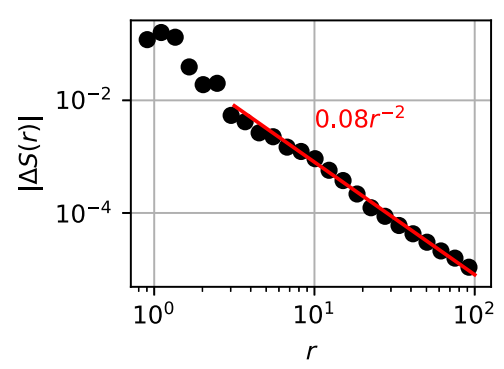

(b)

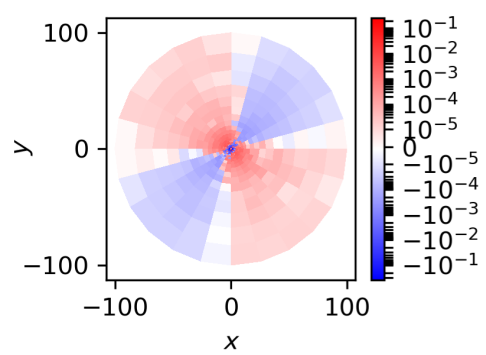

(c)

FIG. 6. (a) Mean softness change $\Delta S$ per frame caused by a rearranging particle at the origin. A prediction from Eq. (5) is plotted as red lines. Here we also plot the volumetric strain $k(r)$ for comparison. Similar to Fig. 3, solid dots and solid lines represent positive values, while hollow circles and dotted lines represent negative values. (b) Same as (a), but for its absolute value. (c) Mean softness change with directional dependence shown. 


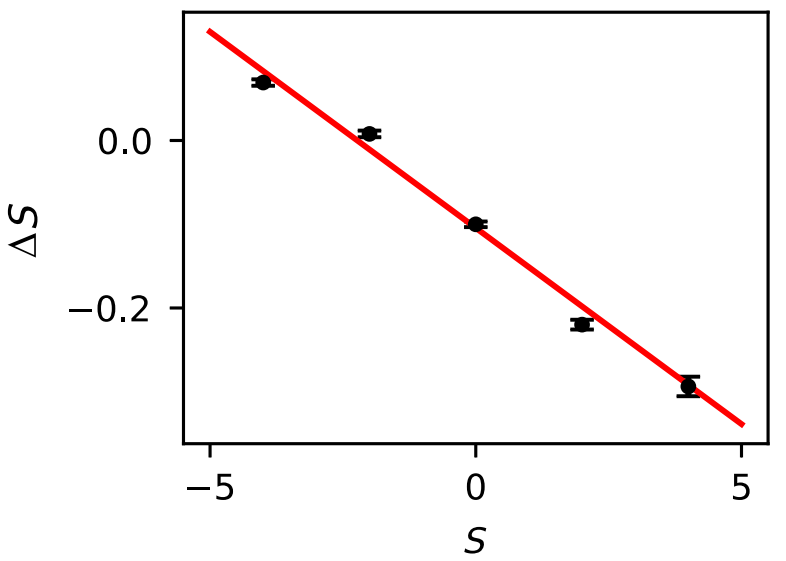

(a)

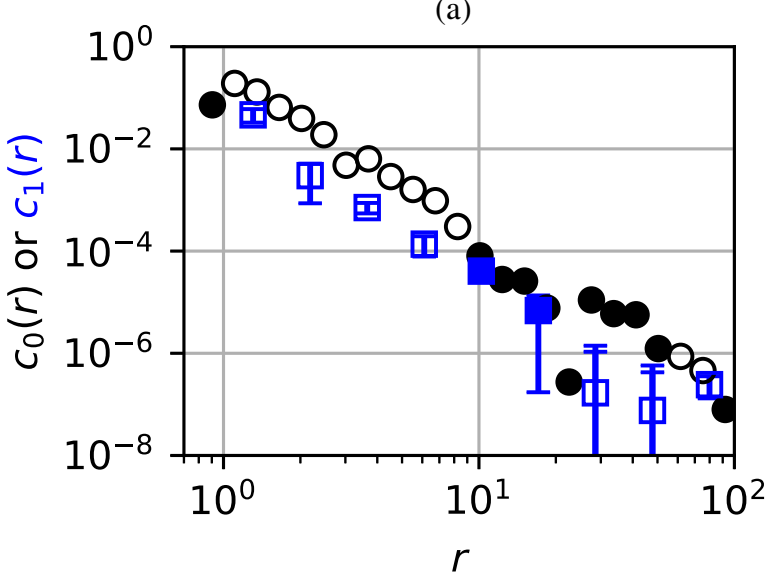

(b)

FIG. 7. (a) Mean softness change $\Delta S$ per frame for a particle with a given $S$ within a distance of $r<1.6$ of a rearranger. The red line is the linear fit. (b) The slope of such linear fits $c_{1}$ (squares), as well as $c_{0}$ (circles) defined in Eq. (5), at different distances $r$. Solid symbols represent positive values, while open symbols represent negative values.

yields the red curve in Fig. 6(a). Note that the red curve provides an excellent description of the black points $[\Delta S(r)]$, capturing the sign as well as the magnitude in the far field.

These results justify the arrow connecting "Rearrangements" to "Softness" in Fig. 1(b).

\section{Effect of strain and softness on rearrangements}

We have shown that rearrangements give rise to deviatoric strain that in turn triggers new rearrangements. We have also shown that rearrangers tend to have high softness. Here we examine how $S$ and $\tilde{\epsilon}$ work in tandem to induce rearrangements. When a particle starts rearranging at frame $f$, we rewind $\delta f$ frames to calculate the shear strain exerted on this particle between $f-\delta f$ and $f$, and the softness $S$ at frame $f-\delta f$. As Fig. 8 shows, the amount of shear strain needed to trigger a rearrangement depends strongly on $S$, but only very weakly on $\delta f$. Thus, softer particles require less shear strain to start rearranging (they have lower local yield strains). This is consistent with earlier results in thermal

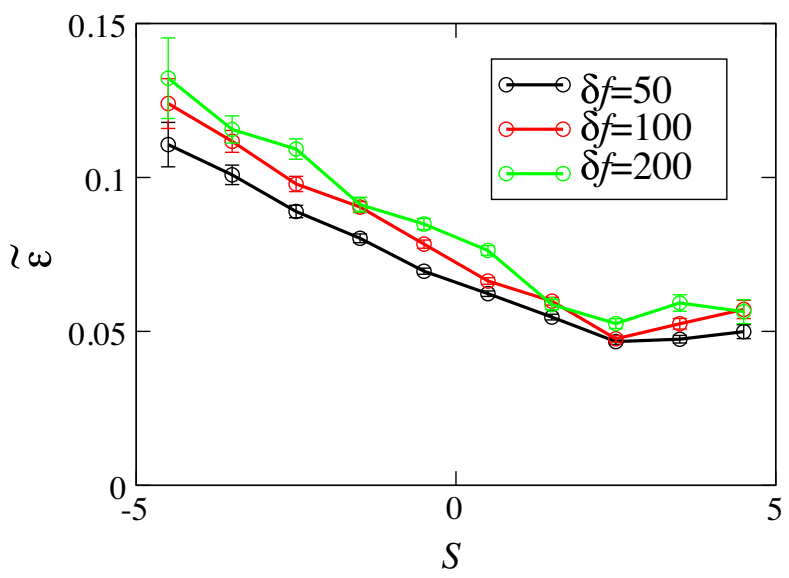

FIG. 8. The amount of shear strain exerted to the local environment of a particle before it starts to rearrange versus the softness of that particle, observed 50, 100, and 200 frames before the rearrangement.

systems that found that softer particles have lower activation energies to rearrange $[20,23]$. Indeed, we have conducted thermal molecular dynamics simulations to find energy barriers comparable to those predicted by Fig. 8 [25].

The results of this section establish that both softness and deviatoric strain are important to trigger future rearrangements, justifying the joining of the arrows connecting "Softness" to "Rearrangement" and "Deviatoric strain" to "Rearrangement." This completes the derivation of Fig. 1(b). Note that we have obtained a quantitative relation for each arrow in the diagram.

\section{DISCUSSION}

In this paper, we study avalanches that occur during energy drops when a two-dimensional jammed binary Hertzian disk packing is sheared quasistatically, using steepest descent to follow the minimization process. We have developed an analysis framework that untangles the interplay of local structure, plastic events, and elasticity. This framework can be applied to any athermal disordered solid under mechanical load as long as the particle positions are tracked with time. Thus, this paper provides a blueprint for constructing structuro-elastoplasticity models that can be applied to a broad class of systems. This includes systems composed of frictional particles and/or particles of complex shape and size distributions. It also includes systems that exhibits shear banding and brittle failure as well as ductile systems. Finally, it can be generalized in any number of spatial dimensions, as we have done to some extent for $d=3$ [25].

The results of our analysis for Hertzian jammed packings are summarized in Fig. 1(b). We expect that the qualitative results of Fig. 1(b) apply quite generally to both two- and three-dimensional ductile disordered solids that exhibit avalanche behavior. We find that (1) a rearrangement alters the softness of a nearby particle according to the difference 
between its softness and the mean softness. This behavior was first observed for 3D Lennard-Jones systems above the glass transition [20], indicating that it is quite general. (2) A rearrangement alters the softness of distant particles through volumetric strain. The existence of a transient volumetric strain, which has not been considered significant, is a feature of elasticity. The fact that local dilation (compaction) increases (decreases) softness is consistent with the previously observed dependence of softness on local density in 3D Lennard-Jones mixtures [20], with the observation that shear bands have reduced local densities in the same system [36], and with our physical understanding of softness, and is therefore also quite general. (3) A rearrangement exerts a deviatoric strain on the rest of the system. This should be generally true for isotropic systems in any dimension. (4) The average yield strain decreases with increasing softness. This is consistent with previous results for 3D Lennard-Jones simulations [20], 2D colloidal glass experiments [37], and 3D aluminum polycrystal simulations [23], showing that the energy barrier for rearrangements decreases with increasing softness.

Figure 1(b) can be viewed as a structuro-elastoplastic model that builds upon earlier elastoplastic models. Our results show that it is essential for the model to include the full tensorial strain induced by a rearrangement as well as a variable to characterize structure. Accordingly, our STEP model includes the distinct effects of both volumetric and deviatoric strain and incorporates structure through softness, which evolves dynamically due to rearrangements.

Note that we find that rearrangements are triggered by deviatoric rather than shear strain indicating shear strain in any direction due to a rearrangement can trigger the next rearrangement equally well. Elastoplastic models typically focus on the component of the local shear strain with the same orientation as the global shear strain $[12,38]$. At least for ductile systems, which do not build up much strain in the direction of applied strain, this assumption misses important physics. More significantly, we have elucidated how the local structural environment of a particle affects and is affected by rearrangements and strain.

It is important to note that there are additional contributions to the interplay between softness, strain, and rearrangers that are not included in Fig. 1(b). For example, softness should affect the strain field caused by a rearranger, since softer regions intuitively should have lower elastic moduli. We have shown that on average the strain field is well described by continuum elasticity, but there are fluctuations around this average strain response that we have not treated here. As another example, not only volumetric strain but also deviatoric strain affects softness, as reported in Ref. [1]. However, we find that the former effect is dominant, which is not surprising since softness depends sensitively on density. We find that the rearranger pair correlation function is isotropic, which suggests that the deviatoric strain is the main contributor in triggering rearrangements, but the volumetric strain is theoretically also capable of triggering rearrangements. Also the $x y$ strain may be important in more brittle systems where the global strain can accumulate by a significant amount. Figure 1(b) should therefore be viewed as a summary of the leading effects that should be included in a structuroelastoplasticity model for the system studied, not as a summary of all the effects that exist. In other words, we have obtained not the complete description of the interplay of softness, rearrangements and elasticity, but a minimal model that includes only the dominant effects.

Our results point to a few factors that may contribute to the ductile behavior observed. First, we find that future rearrangements are triggered by the total deviatoric strain, rather than the $x y$-shear strain that is typically assumed in elastoplastic models. As a result, rearrangements trigger successive rearrangements that are isotropically distributed. In brittle systems, by contrast, the $x y$ strain may well play a more important role since the strain in that direction builds up with relatively few rearrangements or changes of local structure as the system approaches yield [21]. That anisotropy may promote shear band formation by triggering successive rearrangements preferentially in the direction of maximum $x y$ strain.

In addition, a rearranger lowers the softness of nearby particles, discouraging them from rearranging, while on average raising the softness of distant particles, facilitating their rearrangement. Third, rearrangements tend to push the softness of nearby particles toward the mean, which is quite high for the ductile system. Our approach can be applied directly to systems that exhibit shear banding and brittle failure to see whether the interplay is different in such systems. Earlier papers have shown that softness is readily identified in experimental systems for which the positions of particles can be tracked with time [1,39,40]. Our analysis approach for disentangling the interplay of softness, rearrangements, and strain can therefore be applied directly to experiments as well as simulations. It is likely that the key to understanding ductile versus brittle behavior is encapsulated in this interplay.

Besides the brittle-to-ductile transition, many other phenomena in plasticity of disordered solids have also attracted recent attention, for example, power-law distribution of avalanche sizes [9], the ability to reach a steady state under cyclic shear [11], and the discontinuity of the first instability location as a function of the shear orientation [30]. It will be interesting to study the role of local structure in each of these phenomena in future studies.

An important feature of our approach is that it is built on a machine-learned structural quantity, softness. However, many different predictors of rearrangements have recently been tested for two different Lennard-Jones systems, each prepared with two different protocols [21]. In principle our approach could be used for any of the predictors evaluated in Ref. [21], subject to practical constraints. Among the 
predictors, softness has the advantages of excellent scalability $[\mathcal{O}(N)]$, high performance in prediction of rearrangements [21], the lack of need to specify the interaction potential, and easy generalization to wider class of systems, including ones that lack spherically symmetric potentials $[40,41]$. Softness can also readily be generalized to higher spatial dimensions [42].

An alternate theoretical approach has been to view shear bands as associated with critical phase transitions such as the random-field Ising transition [14,43]. The kinetics of such transitions can also involve avalanches, but the underlying mechanisms are somewhat different; for example, elasticity does not mediate the triggering of avalanches in the randomfield Ising model while it is well recognized to play an important role in avalanches of ductile disordered solids.

For over a century, statistical mechanics has served as an extremely powerful tool for dimensional reduction, distilling overwhelming amounts of microscopic information into distributions of one or a few relevant microscopic variables in order to uncover the microscopic origins of macroscopic, collective behavior. However, nonlinear, farfrom-equilibrium phenomena such as plasticity in disordered solids have posed a long-standing challenge to statistical mechanics. In this paper, we have harnessed the power of machine learning for dimensional reduction to identify softness, along with $D_{\min }^{2}$, as two relevant microscopic variables on which to construct a theory of plasticity. Furthermore, we untangled the interplay of softness, $D_{\mathrm{min}}^{2}$ and elasticity to accomplish what statistical mechanics is designed to do-to bridge the gap between microscopic particle-level physics and macroscopic emergent behavior (plasticity). We anticipate that our use of a machine-learned quantity as the basis of a theoretical approach to collective behavior is a harbinger of future research exploiting machine learning to develop theories of particularly thorny many-body physics problems.

\section{ACKNOWLEDGMENTS}

We thank Douglas J. Durian, Robert J. S. Ivancic, Francois Landes, Robert A. Riggleman, Hongyi Xiao, Menachem Stern, and Sam Dillavou for helpful discussions and their careful reading of the manuscript. This work was supported by the UPenn MRSEC NSF-DMR-1720530 (G. Z.), the Simons Foundation for the collaboration "Cracking the Glass Problem" Grant No. 454945 to A. J. L. (S. R. and A. J. L.), and Investigator Grant No. 327939 (A. J. L.), and NSERC via a PGS-D fellowship (S. R.).

\section{APPENDIX A: CONTINUUM-ELASTIC PREDICTIONS FOR STRAIN FIELD INDUCED BY A REARRANGEMENT}

The far field of rearrangement events has long been modeled as that of an Eshelby inclusion, which is the elastic response to a point strain source [29].

Elastoplastic models typically only consider $\sigma_{x y}$, use an elastic kernel which assumes the medium to be incompressible, and take the limit of infinite time (mechanical equilibrium). Since we are interested in understanding the course of avalanches during steepest descent, we need the kernel at finite times with overdamped dynamics. We sketch below the derivation of all components of the continuum strain field.

We begin by considering an infinite elastic medium subject to a point force turning on at $t=0$ at the origin.

We wish to find $G_{i k}(\mathbf{r}, t)$ such that

$$
C_{i p j m} \frac{\partial^{2} G_{j k}}{\partial x_{p} \partial x_{m}}-\eta \frac{\partial G_{i k}}{\partial t}+\delta_{i k} \delta(\mathbf{r}) \Theta(t)=0
$$

Taking a Fourier transform in space and a Laplace transform in time gives us

$$
\begin{aligned}
\tilde{G}_{i k} & =\frac{1}{s}\left[C_{k p i m} q_{p} q_{m}+\eta s \delta_{i k}\right]^{-1} \\
& =\frac{1}{s}\left[\frac{1}{\mu q^{2}+\eta s} \hat{t}_{i} \hat{t}_{k}+\frac{1}{(\lambda+2 \mu) q^{2}+\eta s} \hat{q}_{i} \hat{q}_{k}\right],
\end{aligned}
$$

with the last equality holding for an isotropic medium in 2D. Here $\hat{t}$ is the vector normal to $\hat{q}$.

We invert the spatial Fourier transform, and then the Laplace transform. The result is

$$
\begin{aligned}
G_{i k}= & \frac{1}{8 \pi}\left[\frac{1}{\mu} \Gamma\left(0, \frac{\eta r^{2}}{4 \mu t}\right)+\frac{1}{2 \mu+\lambda} \Gamma\left(0, \frac{\eta r^{2}}{4(\lambda+2 \mu) t}\right)+\frac{4 t}{\eta r^{2}}\left(e^{-\eta r^{2} / 4 \mu t}-e^{-\eta r^{2} / 4(2 \mu+\lambda) t}\right)\right] \delta_{i k} \\
& +\frac{t}{\pi \eta r^{2}}\left(e^{-\eta r^{2} / 4(2 \mu+\lambda) t}-e^{-\eta r^{2} / 4 \mu t}\right) \hat{r}_{i} \hat{r}_{k},
\end{aligned}
$$

where $\Gamma(0, x) \equiv \int_{x}^{\infty} d s s^{-1} e^{-s}$ is the incomplete gamma function (in this case, also the exponential integral function).

Differentiating this twice and symmetrizing over one of the indices allows us to compute $G_{i j k l}$, the strain response to a dipole of force. 
We obtain

$$
\begin{aligned}
G_{i j k l}= & \frac{1}{4 \pi r^{2}}\left[\frac{1}{\mu} e^{-\eta r^{2} / 4 \mu t}+\frac{4 t}{\eta r^{2}}\left(e^{-\eta r^{2} / 4 \mu t}-e^{\left.-\eta r^{2} / 4(2 \mu+\lambda) t\right)}\right]\left[\delta_{i l} \delta_{j k}+\delta_{j l} \delta_{i k}\right]\right. \\
& -\frac{1}{4 \pi r^{2}}\left[\frac{16 t}{\eta r^{2}}\left(e^{-\eta r^{2} / 4 \mu t}-e^{-\eta r^{2} / 4(2 \mu+\lambda) t}\right)+\left(\frac{2}{\mu} e^{-\eta r^{2} / 4 \mu t}-\frac{2}{2 \mu+\lambda} e^{-\eta r^{2} / 4(2 \mu+\lambda) t}\right)\right]\left[\hat{r}_{k} \hat{r}_{l} \delta_{i j}+\hat{r}_{i} \hat{r}_{j} \delta_{k l}+\hat{r}_{i} \hat{r}_{k} \delta_{j l}+\hat{r}_{j} \hat{r}_{k} \delta_{i l}\right] \\
& +\frac{1}{\pi r^{2}}\left[\frac{1}{\mu}\left(4+\frac{\eta r^{2}}{4 \mu t}+\frac{24 \mu t}{\eta r^{2}}\right) e^{-\eta r^{2} / 4 \mu t}-\frac{1}{2 \mu+\lambda}\left(4+\frac{\eta r^{2}}{4(2 \mu+\lambda) t}+\frac{24(2 \mu+\lambda) t}{\eta r^{2}}\right) e^{-\eta r^{2} / 4(2 \mu+\lambda) t}\right] \hat{r}_{i} \hat{r}_{j} \hat{r}_{k} \hat{r}_{l} \\
& -\frac{1}{4 \pi r^{2}}\left[\left(\frac{1}{\mu}+\frac{\eta r^{2}}{2 \mu t}\right) e^{-\eta r^{2} / 4 \mu t}-\frac{2}{2 \mu+\lambda} e^{-\eta r^{2} / 4(2 \mu+\lambda) t}+\frac{16 t}{\eta r^{2}}\left(e^{-\eta r^{2} / 4 \mu t}-e^{\left.-\eta r^{2} / 4(\mu+\lambda) t\right)}\right]\left[\hat{r}_{j} \hat{r}_{l} \delta_{i k}+\hat{r}_{i} \hat{r}_{l} \delta_{j k}\right]\right. \\
& +\frac{t}{2 \pi \eta r^{4}}\left(e^{-\eta r^{2} / 4 \mu t}-e^{-\eta r^{2} / 4(2 \mu+\lambda) t}\right) \delta_{i j} \delta_{k l} .
\end{aligned}
$$

Following previous work, a dipole of $x y$-shear strain at the origin is equivalent to a pair of force dipoles [29]. Assuming this source gives us the elastic strain field [now written in terms of the Poisson ratio $\nu$ and the "diffusion constants" $D_{T} \equiv(\mu / \eta)$ and $\left.D_{L} \equiv(\lambda+2 \mu / \eta)=2 D_{T} /(1-\nu)\right]$ :

$$
\begin{gathered}
k^{(S)}(\mathbf{r}, t)=-\frac{(1-\nu) \sin 2 \theta}{8 \pi r^{2}} e^{-r^{2} / 4 D_{L} t}\left(1+\frac{r^{2}}{4 D_{L} t}\right), \\
\epsilon_{x y}^{(S)}(\mathbf{r}, t)=\frac{\cos 4 \theta}{2 \pi r^{2}}\left[(1-\nu) e^{-r^{2} / 4 D_{L} t}\left(2+\frac{r^{2}}{8 D_{L} t}+\frac{12 D_{L} t}{r^{2}}\right)-e^{-r^{2} / 4 D_{T} t}\left(4+\frac{r^{2}}{4 D_{T} t}+\frac{24 D_{T} t}{r^{2}}\right)\right] \\
-\frac{1}{2 \pi r^{2}}\left(\frac{r^{2}}{4 D_{L} t} e^{-r^{2} / 4 D_{L} t}+\frac{r^{2}}{4 D_{T} t} e^{-r^{2} / 4 D_{T} t}\right), \\
\frac{1}{2}\left[\epsilon_{x x}^{(S)}(\mathbf{r}, t)-\epsilon_{y y}^{(S)}(\mathbf{r}, t)\right]=\frac{\sin 4 \theta}{2 \pi r^{2}}\left[e^{-r^{2} / 4 D_{T} t}\left(4+\frac{r^{2}}{4 D_{T} t}+\frac{24 D_{T} t}{r^{2}}\right)-(1-\nu) e^{-r^{2} / 4 D_{L} t}\left(2+\frac{r^{2}}{8 D_{L} t}+\frac{12 D_{L} t}{r^{2}}\right)\right] .
\end{gathered}
$$

The familiar power-law dependences from elastic equilibrium are realized in the large-time limit:

$$
\begin{aligned}
k^{(S)}(\mathbf{r}, \infty) & =\frac{(\nu-1) \sin 2 \theta}{2 \pi r^{2}}, \\
\epsilon_{x y}^{(S)}(\mathbf{r}, \infty) & =\frac{(1+\nu) \cos 4 \theta}{2 \pi r^{2}}, \\
\frac{1}{2}\left[\epsilon_{x x}^{(S)}(\mathbf{r}, \infty)-\epsilon_{y y}^{(S)}(\mathbf{r}, \infty)\right] & =-\frac{(1+\nu) \sin 4 \theta}{2 \pi r^{2}} .
\end{aligned}
$$

These results together explain why the volumetric strain is observed to have a $\sin (2 \theta)$ dependence, and why the deviatoric strain magnitude is isotropic.

Notice, however, that $\int d \theta k(r, \theta, t)=0$ for such a shear strain source. To explain the apparent nonzero value of $\int d \theta k(r, \theta, t)$ for short times in our simulations, we must consider the effect of a transient expansion source. The local region surrounding a rearrangement might be expected, on average, to have a different volume than in the initial state.
In an infinite system, the kernel above gives for a point plastic compression at the origin:

$$
k^{(C)}(\mathbf{r}, t)=\frac{1+\nu}{2} \frac{1}{4 \pi D_{L} t} e^{-r^{2} / 4 D_{L} t} .
$$

As long as the Poisson ratio is close to 1 , this precisely conserves volume in an infinite system, when added to the point compression at the origin.

We expect that since our system is finite (and the shorttime Poisson ratio is far from 1), this kernel would need to be modified near the boundaries of the system to satisfy the periodic boundary conditions and conserve the total volume. We find that it works adequately for the bulk for our data however, and our data at $r$ close to the box size are difficult to resolve-we have chosen the $y$ range in the top left-hand box of Fig. 3 to exclude points beyond $r=30$ because the error bars are comparable to the absolute value.

The full response to a given event will be a sum of the responses to strain [Eq. (A8)] and compression sources [Eq. (A9)] with appropriate prefactors, although for 
measurements where its contribution is nonzero we expect the strain source to be dominant.

\section{APPENDIX B: COMPARISON OF ANALYTICAL AND NUMERICAL $k(r), \tilde{\epsilon}$, and $\epsilon_{x y}$ RESULTS}

Since we have derived analytical formulas for the strain, Eqs. (A8) and (A9), we can make a comparison with our numerical results. We have numerically measured instantaneous elastic constants $\lambda+2 \mu=0.3533$ and $\nu=0.3408$ for our system by applying a small $\left(10^{-6}\right)$ strain on the simulation box and measuring the force.

The time interval between frames $t$ is not fixed since we record frames that are equidistant in configuration space; see the Supplemental Material [25]. We plot the distribution of times between frames in Fig. S1, and find that the most probable time interval is $t \approx 100$. The definition of our time implies that $\eta=1$. With these parameters, Eq. (A9) predicts a Gaussian that decays to $0.1 \%$ of its peak height at $r=31$, roughly consistent with the actual result presented in Fig. 3.

For the total deviatoric strain $\tilde{\epsilon}$ and $x y$ strain $\epsilon_{x y}$, we have numerically confirmed that they decay as power laws: $\tilde{e}=$ $\tilde{c} / r^{2}$ and $\epsilon_{x y}=c_{x y} / r^{2}$ (Fig. 3), which matches the prediction in Eq. (A8). The prefactors, i.e., constants $\tilde{c}$ and $c_{x y}$, were not predicted in Appendix A since our theory does not take into consideration the average amount of plastic strain caused by a rearranger.

Nevertheless, we can approximately measure this quantity. The strains in Eq. (A8) are for a plastic strain $\epsilon_{x y}^{\mathrm{pl}}=\epsilon_{0} A \delta(\mathbf{r})$; i.e., the prefactor of the far-field strain is equal to the product of the area $A=\pi r_{D}^{2}$ of the rearrangement and its plastic strain.

If the rearrangements have a distribution of plastic strains $\epsilon_{0}$ and orientations $\theta^{\prime}$, then by rotating the kernel and assuming the distribution of $\theta^{\prime}$ is even we find that

$$
\begin{gathered}
\langle\tilde{\epsilon}(r)\rangle=\frac{1+\nu}{2} \frac{r_{D}^{2}\left\langle\tilde{\epsilon_{0}}\right\rangle}{r^{2}}, \\
\left\langle\epsilon_{x y}(r)\right\rangle=\frac{1+\nu}{2} r_{D}^{2}\left\langle\epsilon_{0, x y} \cos \left(4 \theta^{\prime}\right)\right\rangle \frac{\cos (4 \theta)}{r^{2}}, \\
\langle k(r)\rangle=\frac{\nu-1}{2} r_{D}^{2}\left\langle\epsilon_{0, x y} \cos \left(2 \theta^{\prime}\right)\right\rangle \frac{\sin (2 \theta)}{r^{2}} .
\end{gathered}
$$

We will neglect the $\cos 4 \theta^{\prime}$ and $\cos 2 \theta^{\prime}$ in our rough estimates.

We find that the $D_{\min }^{2}$ correlation length [1] is $r_{D}=3.6$; i.e., the correlation between $D_{\min }^{2}(\mathbf{0})$ and $D_{\min }^{2}(\mathbf{r})$ is approximately $\exp (-|\mathbf{r}| / 3.6)$ for small $|\mathbf{r}|$. The area of the event is then estimated as $\pi r_{D}^{2}$. We then calculate the local-fit deviatoric and $x y$ strain within a radius of $r_{D}$ around each rearranger, and find on average $\tilde{\epsilon}=3.6 \times 10^{-3}$ and $\epsilon_{x y}=$ $1.8 \times 10^{-4}$ at the rearranging site. Theoretically, this predicts that the prefactors are $\tilde{c}=(1+\nu / 2) \tilde{\epsilon} r_{D}^{2}=0.031$,
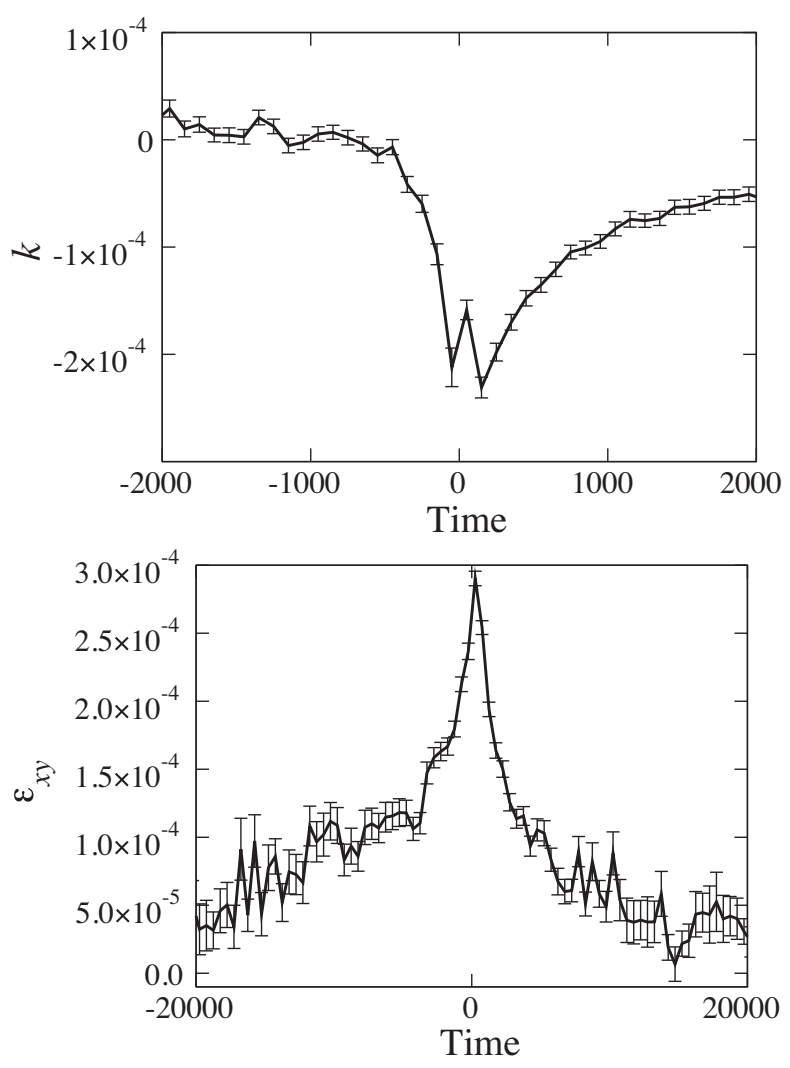

FIG. 9. (Top) The local-fit volumetric strain of a rearranger versus time. To average over different rearranging events, they are temporally aligned so that they start $\left(D_{\min }^{2}\right.$ rises above the threshold) at $t=0$. Rearrangements usually end at some time $t$ between $10^{2}$ and $10^{3}$. (Bottom) Same as left, except for local-fit $x y$-shear strain.

$c_{x y}=(1+\nu / 2) \epsilon_{x y} r_{D}^{2}\langle|\cos 4 \theta|\rangle=1.0 \times 10^{-3}$, and $c_{k}=$ $(1-\nu / 2) \epsilon_{x y} r_{D}^{2}\langle|\sin 2 \theta|\rangle=5.0 \times 10^{-4}$. This roughly matches the fits presented in Fig. 2 of the main text, which have $\tilde{c}=0.03, c_{x y}=1.5 \times 10^{-3}$, and $c_{k}=5.0 \times 10^{-4}$.

Why do our numerical results match the analytical derivations for shear strains produced by a shear source, Eqs. (A6) and (A7), in the infinite-time limit of Eq. (A8), but match that for the volumetric strain produced by a compression source, Eq. (A9), at a finite time? It turns out that at the rearranging site, the plastic shear occurs over a much longer time interval than the plastic compression. We plot these strain components at the rearranging site versus time in Fig. 9. If we approximate such strain-time curves with Gaussians, then the numerically measured strain at distance $r$ should be the convolution of previously derived finite-time analytical result and Gaussians, i.e.,

$$
\begin{gathered}
k(r, \text { numerical })=c_{k} \int_{-\infty}^{0} \exp \left(-\alpha t^{2}\right) k\left(r, t-t^{\prime}\right) d t^{\prime}, \\
\epsilon_{x y}(r, \text { numerical })=c_{x y} \int_{-\infty}^{0} \exp \left(-\beta t^{2}\right) \epsilon_{x y}\left(r, t-t^{\prime}\right) d t^{\prime},
\end{gathered}
$$


where $k(r, t)$ and $\epsilon_{x y}(r, t)$ are given in Eqs. (A9) and (A6), respectively. We numerically compute these integrals for various parameters. For $k$, the integral fits numerical data well at $\alpha=6.1197 \times 10^{-5}$, as shown in Fig. 3. This indicates that the width of the Gaussian is about $\alpha^{-1 / 2}=127.83$, roughly consistent with Fig. 9. For $\epsilon_{x y}$, however, it turns out that Eq. (B4) cannot closely fit our numerical result, which decays slightly slower than $r^{-2}$ (Fig. 3). No matter how small $\beta$ is, Eq. (B4) gives an $\epsilon_{x y}$ that decays slightly faster than $r^{-2}$. We see two possible reasons for this difference: (1) a finite size effect as $r$ becomes comparable to the box size or (2) the interference between simultaneous rearrangements in our numerical results. As we discuss in Sec. II B, we filter out frames with multiple rearrangements, but such filtration cannot be perfect.

[1] E. D. Cubuk, R. Ivancic, S. S. Schoenholz, D. Strickland, A. Basu, Z. Davidson, J. Fontaine, J. L. Hor, Y.-R. Huang, Y. Jiang et al., Structure-Property Relationships from Universal Signatures of Plasticity in Disordered Solids, Science 358, 1033 (2017).

[2] D. J. Durian, Foam Mechanics at the Bubble Scale, Phys. Rev. Lett. 75, 4780 (1995).

[3] J. P. Sethna, K. A. Dahmen, and C. R. Myers, Crackling Noise, Nature (London) 410, 242 (2001).

[4] E. K. H. Salje and K.A. Dahmen, Crackling Noise in Disordered Mateirals, Annu. Rev. Condens. Matter Phys. 5, 233 (2014).

[5] J. P. Sethna, M. K. Bierbaum, K. A. Dahmen, C. P. Goodrich, J. R. Greer, L. X. Hayden, J. P. Kent-Dobias, E. D. Lee, D. B. Liarte, X. Ni et al., Deformation of Crystals: Connections with Statistical Physics, Annu. Rev. Mater. Res. 47, 217 (2017).

[6] K. A. Dahmen, J. T. Uhl, and W. J. Wright, Why the Crackling Deformations of Single Crystals, Metallic Glasses, Rock, Granular Materials, and the Earth's Crust Are So Surprisingly Similar, Front. Phys. 7, 176 (2019).

[7] R. Conner, W. L. Johnson, N. Paton, and W. Nix, Shear Bands and Cracking of Metallic Glass Plates in Bending, J. Appl. Phys. 94, 904 (2003).

[8] S. Karmakar, E. Lerner, and I. Procaccia, Statistical Physics of the Yielding Transition in Amorphous Solids, Phys. Rev. E 82, 055103(R) (2010).

[9] J. Barés, D. Wang, D. Wang, T. Bertrand, C. S. O'Hern, and R. P. Behringer, Local and Global Avalanches in a TwoDimensional Sheared Granular Medium, Phys. Rev. E 96, 052902 (2017).

[10] Z. Budrikis, D. F. Castellanos, S. Sandfeld, M. Zaiser, and S. Zapperi, Universal Features of Amorphous Plasticity, Nat. Commun. 8, 15928 (2017).

[11] M. M. Bandi, H. G. E. Hentschel, I. Procaccia, S. Roy, and J. Zylberg, Training, Memory and Universal Scaling in Amorphous Frictional Granular Matter, Europhys. Lett. 122, 38003 (2018).
[12] A. Nicolas, E. E. Ferrero, K. Martens, and J.-L. Barrat, Deformation and Flow of Amorphous Solids: Insights from Elastoplastic Models, Rev. Mod. Phys. 90, 045006 (2018).

[13] A. Shavit and R. A. Riggleman, Strain Localization in Glassy Polymers under Cylindrical Confinement, Phys. Chem. Chem. Phys. 16, 10301 (2014).

[14] M. Ozawa, L. Berthier, G. Biroli, A. Rosso, and G. Tarjus, Random Critical Point Separates Brittle and Ductile Yielding Transitions in Amorphous Materials, Proc. Natl. Acad. Sci. U.S.A. 115, 6656 (2018).

[15] M. Popović, T. W. J. de Geus, and M. Wyart, Elastoplastic Description of Sudden Failure in Athermal Amorphous Materials during Quasistatic Loading, Phys. Rev. E 98, 040901(R) (2018).

[16] F. Spaepen, A Microscopic Mechanism for Steady State Inhomogeneous Flow in Metallic Glasses, Acta Metall. 25, 407 (1977).

[17] M. L. Falk and J. S. Langer, Deformation and Failure of Amorphous, Solidlike Materials, Annu. Rev. Condens. Matter Phys. 2, 353 (2011).

[18] A. Widmer-Cooper, H. Perry, P. Harrowell, and D. R. Reichman, Irreversible Reorganization in a Supercooled Liquid Originates from Localized Soft Modes, Nat. Phys. 4, 711 (2008).

[19] M. L. Manning and A. J. Liu, Vibrational Modes Identify Soft Spots in a Sheared Disordered Packing, Phys. Rev. Lett. 107, 108302 (2011).

[20] S. S. Schoenholz, E. D. Cubuk, D. M. Sussman, E. Kaxiras, and A.J. Liu, A Structural Approach to Relaxation in Glassy Liquids, Nat. Phys. 12, 469 (2016).

[21] D. Richard, M. Ozawa, S. Patinet, E. Stanifer, B. Shang, S. A. Ridout, B. Xu, G. Zhang, P. K. Morse, J.-L. Barrat et al., Predicting Plasticity in Disordered Solids from Structural Indicators, Phys. Rev. Mater. 4, 113609 (2020).

[22] S. S. Schoenholz, E. D. Cubuk, E. Kaxiras, and A. J. Liu, Relationship between Local Structure and Relaxation in Out-of-Equilibrium Glassy Systems, Proc. Natl. Acad. Sci. U.S.A. 114, 263 (2017).

[23] T. A. Sharp, S. L. Thomas, E. D. Cubuk, S. S. Schoenholz, D. J. Srolovitz, and A. J. Liu, Machine Learning Determination of Atomic Dynamics at Grain Boundaries, Proc. Natl. Acad. Sci. U.S.A. 115, 10943 (2018).

[24] D. M. Sussman, S. S. Schoenholz, E. D. Cubuk, and A. J. Liu, Disconnecting Structure and Dynamics in Glassy Thin Films, Proc. Natl. Acad. Sci. U.S.A. 114, 10601 (2017).

[25] See Supplemental Material at http://link.aps.org/ supplemental/10.1103/PhysRevX.11.041019 a video of an avalanche, details of our energy-minimization and sampling schemes, parameters for training, softness distribution breakdown by coordination numbers, rearranging probability at a positive temperature, and results in $3 \mathrm{D}$.

[26] C. E. Maloney and A. Lemaître, Amorphous Systems in Athermal, Quasistatic Shear, Phys. Rev. E 74, 016118 (2006).

[27] M. L. Falk and J. S. Langer, Dynamics of Viscoplastic Deformation in Amorphous Solids, Phys. Rev. E 57, 7192 (1998).

[28] J. Behler and M. Parrinello, Generalized Neural-Network Representation of High-Dimensional Potential-Energy Surfaces, Phys. Rev. Lett. 98, 146401 (2007). 
[29] G. Picard, A. Ajdari, F. Lequeux, and L. Bocquet, Elastic Consequences of a Single Plastic Event: A Step towards the Microscopic Modeling of the Flow of Yield Stress Fluids, Eur. Phys. J. E 15, 371 (2004).

[30] O. Gendelman, P. K. Jaiswal, I. Procaccia, B. S. Gupta, and J. Zylberg, Shear Transformation Zones: State Determined or Protocol Dependent?, Europhys. Lett. 109, 16002 (2015).

[31] K. E. Jensen, D. A. Weitz, and F. Spaepen, Local Shear Transformations in Deformed and Quiescent Hard-Sphere Colloidal Glasses, Phys. Rev. E 90, 042305 (2014).

[32] A. Le Bouil, A. Amon, S. McNamara, and J. Crassous, Emergence of Cooperativity in Plasticity of Soft Glassy Materials, Phys. Rev. Lett. 112, 246001 (2014).

[33] S. Karmakar, E. Lerner, and I. Procaccia, Statistical Physics of the Yielding Transition in Amorphous Solids, Phys. Rev. E 82, 055103(R) (2010).

[34] A. Barbot, M. Lerbinger, A. Hernandez-Garcia, R. GarcíaGarcía, M. L. Falk, D. Vandembroucq, and S. Patinet, Local Yield Stress Statistics in Model Amorphous Solids, Phys. Rev. E 97, 033001 (2018).

[35] D. Rodney, A. Tanguy, and D. Vandembroucq, Modeling the Mechanics of Amorphous Solids at Different Length Scale and Time Scale, Model. Simul. Mater. Sci. Eng. 19, 083001 (2011).

[36] A. D. S. Parmar, S. Kumar, and S. Sastry, Strain Localization Above the Yielding Point in Cyclically Deformed Glasses, Phys. Rev. X 9, 021018 (2019).
[37] X. Ma, Z.S. Davidson, T. Still, R. J. S. Ivancic, S. S. Schoenholz, A.J. Liu, and A. G. Yodh, Heterogeneous Activation, Local Structure, and Softness in Supercooled Colloidal Liquids, Phys. Rev. Lett. 122, 028001 (2019).

[38] Z. Budrikis and S. Zapperi, Avalanche Localization and Crossover Scaling in Amorphous Plasticity, Phys. Rev. E 88, 062403 (2013).

[39] E. D. Cubuk, S. S. Schoenholz, J. M. Rieser, B. D. Malone, J. Rottler, D. J. Durian, E. Kaxiras, and A. J. Liu, Identifying Structural Flow Defects in Disordered Solids Using Machine-Learning Methods, Phys. Rev. Lett. 114, 108001 (2015).

[40] M. Harrington, A. J. Liu, and D. J. Durian, Machine Learning Characterization of Structural Defects in Amorphous Packings of Dimers and Ellipses, Phys. Rev. E 99, 022903 (2019).

[41] E. D. Cubuk, A. J. Liu, E. Kaxiras, and S. S. Schoenholz, Unifying Framework for Strong and Fragile Liquids via Machine Learning: A Study of Liquid Silica, arXiv: 2008.09681.

[42] S. A. Ridout, J. W. Rocks, and A. J. Liu, Correlation of Plastic Events with Local Structure in Jammed Packings across Spatial Dimensions, arXiv:2011.13049.

[43] G. Parisi, I. Procaccia, C. Rainone, and M. Singh, Shear Bands as Manifestation of a Criticality in Yielding Amorphous Solids, Proc. Natl. Acad. Sci. U.S.A. 114, 5577 (2017). 